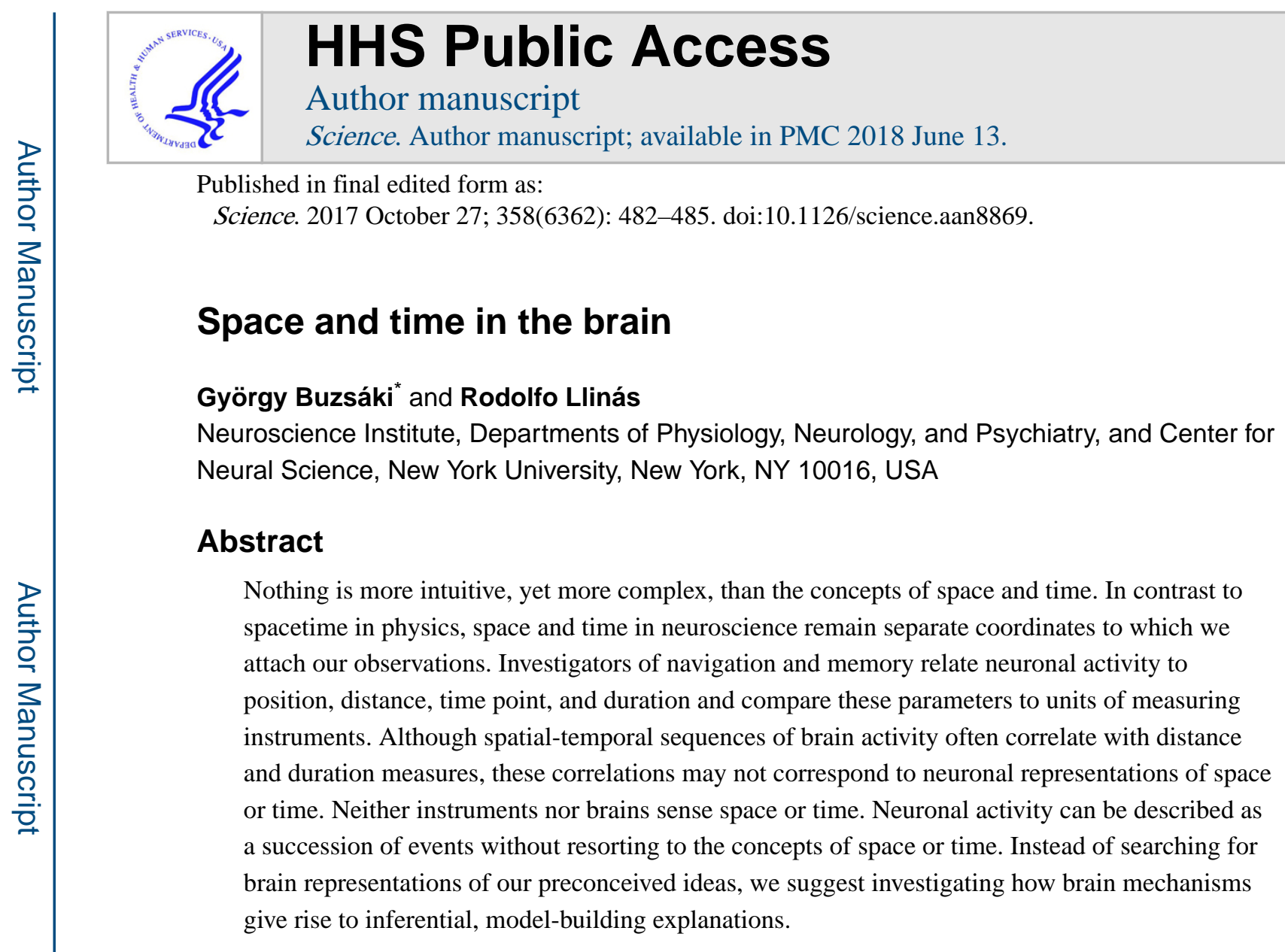

For most cultures, space and time are used to map and explain the vastness and complexities of the universe. These terms are often used interchangeably-for instance, "The Iroquois live 2 days from us." Linguists note that most temporal words have a spatial sense as their primary meaning (1): Half of the world's languages do not have grammatical tense to specify past or future. The Amondawa in the Amazon and the Aborigines of inner Australia do not conceive of time as something independent of other things or something in which events occur. Yet these cultures understand ordering, sequences of events, and relationships $(2,3)$. Thus, it is not obvious that space and time are universal and independent.

Modern science has radically transformed these dimensionless concepts with the introduction of measuring instruments. Space and time were replaced with their definable variants: (i) distance and displacement and (ii) duration and interval, which were quantified by the units of human-made instruments, such as rulers and clocks, thereby giving them practical meanings. In classical physics, the "theater" or "container" metaphor of space and time determines the exact location and speed of a particle. Distance and duration are equated via velocity. Research in neuroscience continues to be performed within this framework of classical physics (4), even though in contemporary physics "there is no longer space which 'contains' the world, and there is no time 'in which' events occur" (5). In this Review, we summarize current neuroscience views on space and time, discuss whether the brain perceives or makes distance and duration, analyze how assumed representations of distance

“Corresponding author. gyorgy.buzsaki@nyumc.org. 
and durations relate to each other, and consider the option that space and time are mental constructs.

\section{Representation of space in the brain}

Extensive studies have separately examined the brain mechanisms of representing space and time. A common philosophy in most of these studies is that space and time are preexisting categories; therefore, the research goal is to understand how we sense them. In addition to distance and duration, new questions have been posed: "Where am I?" (position) and "What time is it?" (a point in time or "now").

Not surprisingly, the field of neuroscience began to define space from a sensory perspective and postulated numerous spaces - such as palm space, oral space, body space, visuo-ocular space, and instrumental space-first based on investigating brain-damaged patients (6). For example, when an Italian patient who had suffered a right parietal stroke was asked to imagine facing the Piazza Del Duomo in Milan and to describe the scene, he correctly identified buildings on his right but omitted those on the left. When asked to imagine standing at the opposite end of the Piazza, the buildings he listed were on the other, previously neglected, side, which was now to his right (7). Such "hemi-neglect" patients can perceive and recall objects per se but cannot describe the objects in their proper spatial relationship or access the contralateral scene from their imagery. Surprisingly, despite this profound deficit, these patients can navigate and find places in a city or at home $(7,8)$.

Animal experiments corroborate the clinical observations. In parietal areas homologous to those whose damage causes hemi-neglect in humans, neuronal populations combine environmental and corporeal inputs. Yet the parietal cortex has no topographic map of the body or the environment. Instead, neurons in this brain region (known as LIP and 7a) integrate multimodal information from scenes to form parsimonious representations described as eye-, head-, or arm-centered coordinates (9). Another region (called MSTd) combines visual motion signals with eye movement and vestibular signals (10) to specify the observer's movement path (11). From such experiments, the notion emerged that the parietal cortex supports mainly body-centered (egocentric) spatial behaviors. Transforming this egocentric coordinate system into a world-centered (allocentric) representation of space has been attributed to the hippocampus-entorhinal cortex system $(10,12,13)$.

Experiments on the hippocampal-entorhinal system in rodents identified mechanisms that define allocentric coordinates similar to those of classical physics. Hippocampal and entorhinal principal neurons have place fields and grid fields, respectively, which collectively generate a spatial map that represents landmarks, objects, and relative locations $(14,15)$. The map is a "three-dimensional Euclidean space...conveying the notion of an allembracing, continuous space" and "a prerequisite to the experiencing of objects and their motions" (14). However, it has remained ambiguous whether the cognitive map theory regards space as real and a priori, which is sensed or represented in the hippocampalentorhinal system, or whether the concept of space is constructed mentally by the brain without any assumption of its existence. 
Establishing a relationship between brain activity and distances can be done by the experimenter, who can relate neuronal recordings to instrument-measured units. However, instrument measurements are not available for neurons for such calibration. Establishing a relationship between neuronal activity and distances in the world requires ambulation, which is supported by a consortium of mechanisms, including optic and haptic flow from local cues, vestibular acceleration signals, and perhaps the counting of steps taken. The combination of distance with head direction information (16) becomes displacement (i.e., a vector). These computations are referred to as path integration (17). Only through such calibration process can the brain acquire meaning of distance and direction. The emerging picture is that of a brain that constructs structured sequences of neuronal cell assemblies whose function is to infer trajectories through the lived or explored world. It should be noted that exploratory experience is not a passive representation or perception of space but a construction of relationships. Yet these formulations of map-based and path integrationbased navigation cannot account for another computation attributed to this system: memory (18).

\section{Episodic memory: Mental travel in space and time}

Navigation and memory are deeply connected. Analogous to map- and path-based navigation, there are two forms of hippocampal system-dependent memories: memorized facts (or semantic memory) and one's personal experiences (episodic memory) (19). To reexperience egocentric episodes, we project ourselves back in space and time (episodic recall) (19), whereas traveling into the imagined future represents planning (prediction). The neuronal mechanisms used to create and recall episodic memory are analogous to those evolved for computing first-order (neighborhood) and higher-order (e.g., shortcuts, detours) distances to explore the physical world via path-based navigation. Similarly, neural algorithms that support map-based navigation are consonant with those needed to create and remember semantic knowledge (20). Like the formation of allocentric maps from egocentric exploration (20), semantic knowledge emerges gradually after repeated encounters with the same thing or event by the episodic memory system $(21,22)$.

This framework implies that most cortical networks have a dual use: environment-dependent and/or internally organized (Fig. 1A). For example, sequential firing of place cells in maze corridors appears to depend on environmental inputs and tracks an animal's progress as it traverses a maze. However, when a rat is trained to run in a wheel or on a treadmill during the delay part of a memory task, neuronal assembly sequences (trajectories) emerge in the hippocampus whose physiological features are difficult to distinguish from the place cell sequences in the maze $(23,24)$. The self-organized neuronal trajectories depend on the integrity of hippocampal theta oscillations (25), are distinctly different after previous left and right corridor choices, and predict the animal's future choice several seconds before its behavioral decision (Fig. 1). Self-organized cell assembly sequences have been also observed in other memory-related systems, including the entorhinal cortex $(26,27)$, the prefrontal cortex (28), and the parietal cortex (29). These findings indicate that neuronal mechanisms associated with navigation and memory are similar: they both establish order relationships (20). However, memory mechanisms are no longer linked to metric distances in the outside world. 
Storing and remembering what happened to us, where and when, defines our personal episodic memories, distinguishing us from others (19). This definition of episodic memory requires neuronal mechanisms to support both the "where" and "when" axes. Many investigators have postulated the existence of neuronal clocks in the brain and assumed that these clocks make time needed for various computational purposes $(23,24,30-33)$. During wheel or treadmill running in a memory task, the ordinal sequences of neurons faithfully track elapsed duration (termed "time cells") (Fig. 1B) from the beginning of the run on subsequent trials in both the hippocampus and the entorhinal cortex $(23,24)$. Because place cell firing frequency varies as a function of running velocity, the elapsed time (and distance) can be continuously derived from the combined knowledge of velocity and sequential firing of neuronal assemblies (34). These experiments demonstrate that sequential neuronal activity can be tightly correlated with instrument-measured units. However, they do not show that these neuronal circuits are dedicated to compute duration per se.

\section{Warping space and time}

Time flies during enjoyable activities but slows when we are bored. Highly motivated states, novel situations, and focused cognitive activity (such as giving a talk) are associated with underestimation of time. Conversely, aversive situations, fatigue, and sleepiness are associated with prolonged subjective time, possibly via affecting dopaminergic signaling (35).

When we type on a keyboard and letters appear on the screen, we feel that we are the agent of our action. If increasing delays are introduced between the keystrokes and the letters, the brain accommodates. But if the artificial delay is suddenly removed, it annoyingly feels that the letters appear before our keystrokes (36). Space and time compression and even time reversal occur with each saccadic eye movement. Objects flashed the saccade are compressed parallel to the path of the saccade (37). Perisaccadic durations are underestimated by approximately the same magnitude as distances $(36,38)$, and study participants often reverse the order of presented events (39). Firing-pattern changes of parietal neurons may underlie the perceived compression of space and time $(40,41)$.

The space-time union of subjective experience is amply demonstrated by asking study participants to imagine themselves as Lilliputians and to engage in activities in scale models $(1 / 6,1 / 12$, and $1 / 24$ scales) of a familiar full-size lounge. Participants were asked to inform the investigator when they subjectively felt that $30 \mathrm{~min}$ had passed. Notably, subjective time acceleration was proportional to the size of the scaled-down models (42).

Warping of distances and durations is typical in hippocampal computation as well. Planning routes from memory involves compression of experienced time, with longer routes and slower movement speeds during the original experience leading to greater compression rates (43). Distances correspond to proportional milliseconds-long intervals within hippocampal theta oscillations (Fig. 2) (24). Even larger time compression of distances occurs when the experienced travel trajectories are replayed during sleep in either a forward or reversed manner (44). Overall, the distance-duration relativity suggests that space and time correspond to the same brain computations. 
In the laboratory, we often find reliable correlations between neuronal activity in various brain regions and succession of events (temporal sequence of representations). However, such relationship does not mean that neuronal activity computes time (representation of temporal sequences) $(45,46)$, even if the succession of events refers to units of clock time. First, neither clocks nor brains make time per se (47). Second, representing or sensing duration implies that time is a preexisting category whose passage the brain detects. However, we do not directly sense time. For example, the amount of light and temperature correlates with the duration of a day, and the brain can track such magnitudes. From the rate of change of these magnitudes, we can mentally construct the concept of the flow of time (45). Clocks have made such inferences ever more precise in modern life.

\section{Ordered neuronal sequences}

Instead of taking space, time, or other mental constructs as a priori variables (i.e., the explananda) and searching for their neuronal mechanisms with expected similar boundaries (i.e., the explanans) (48), we consider a reversed strategy. One way to ground neuronal correlates of distance and duration is to relate them to an animal's actions (49). Navigation -in the real world or the mental domain — can be described by the ordinal succession of events, or "motion" in physics terms. Motion is characterized by velocity and acceleration and has magnitude and direction, which can be sensed by vestibular, proprioceptive, and visual receptors. Magnitude (rate of change, acceleration) and direction are key navigational parameters that are closely related to duration and displacement measures $(50,51)$. As environment-driven functions become internalized in brains of increasing complexity (52), the control of the rate of change may be taken over by attention, in lieu of velocity. Although attention is a hypothetical construct, it has been suggested to affect response magnitude via neuronal gain control (53), similar to velocity (34). This may be the evolutionary route giving rise to the concepts of time and space (49).

Both the parietal cortex and hippocampal-entorhinal system can be considered general purpose sequence generators that continuously tile the gaps between events to be linked: encoding content-limited ordinal structure, thereby referencing to and linking cortical areas where semantic details of the events are processed $(23,54)$. This ordered sequential access to neocortical representations is the physiologic interpretation of episodic memory $(19,21)$. Experimental work supports the ordinal sequence function of the hippocampus. When patients with hippocampal damage were examined after a tour around a campus, they could recall spatial and temporal aspects and details about particular events. However, whereas control participants could well remember the sequential order of the tour events even a month later, the patients' recalled order of events was unrelated to reality (Fig. 3) (55). Hippocampal damage in rats also induces a deficit in learning sequential order of odor stimuli (56). Likewise, the primary purpose of perisaccadic neuronal events is to predict future eye-position sequences (9).

The ordinal sequences in neuronal trajectories can represent the past, present, and future (Fig. 1) (22), providing an explanation for why brain structures and mechanisms associated with memory (postdiction), imagination, and planning (prediction or prospection) overlap 
$(49,57,58)$. Postdiction and prediction may be different names for the same brain computation referenced to a relationship with the current experience (58).

\section{Broader thinking}

The hippocampal-entorhinal system has a topographically organized bidirectional communication with the large neocortex. During the course of mammalian evolution and the corresponding disproportional enlargement of the neocortex, hippocampal inputs shifted from largely sensory and motor representations in the rodent to interactions mainly with other higher-order cortical areas in primates (59). Hippocampal circuits are largely "blind" regarding the modality and nature of cortical inputs. They process the sent messages the same way, regardless of their origin. Given the many possible routes, the answers to the question "What is the function of this circuit?" will be very different, depending on the routes the investigator tests in a given experiment. The function may appear to be space (14, $15)$, time (24), sound frequency (27), odor-sound sequence (60), memory (18), or something else, even though the hippocampus responds to each case by generating ordinal cell assembly sequences relevant to the particular situation (20). These considerations generalize to other brain regions as well $(61,62)$.

Relating sensory inputs to brain activity can provide important yet limited clues to the function of neuronal circuits, and there is no doubt that the terms "space" and "time," as well as other mental constructs, will be part of research for years to come. These concepts are part of our everyday lives. Regarding sequential order as space or time is an attempt to supplement the "outside-in" strategy with a brain-centered, "inside-out" approach. It is such inferential, model-building, explanation-seeking brain mechanisms that should lead our quest for clarifying these fundamental concepts.

\section{Acknowledgments}

We thank A. Berényi, D. Buonomano, O. Devinsky, A. Fernandez-Ruiz, K. Friston, C. Haimerl, D. Levenstein, J. Long, W. Ma, A. Maurer, M. Mehta, H. Rotstein, V. Varga, and the NeuWrite group at New York University for useful feedback on various versions of the manuscript. This work was funded by the NIH (grants MH54671, NS 090583, NS090526, and NS074015), the Simons Foundation, and the Mathers Foundation.

\section{REFERENCES AND NOTES}

1. Traugott, EC. On the expression of spatio-temporal relations in language. In: Greenberg, JH.Ferguson, CA., Moravcsik, EA., editors. Universals of Human Language III. Stanford Univ. Press; 1978. p. 369-400.

2. Sinha C, Da Silva Sinha V, Zinken J, Sampaio W. Lang Cogn. 2011; 3:137-169.

3. Wositsky, J. Born Under the Paperbark Tree: A Man's Life. Harney, YB., editor. JB Books; 1999.

4. Muller, RA. Now: The Physics of Time. W. W. Norton; 2015.

5. Rovelli, C. Reality Is Not What It Seems: The Journey to Quantum Gravity. Penguin Books; 2016.

6. Palliard, J. Motor and representational framing of space. In: Paillard, J., editor. Brain and Space. Oxford Univ. Press; 1991. p. 163-182.

7. Bisiach E, Luzzatti C. Cortex. 1978; 14:129-133. [PubMed: 16295118]

8. van den Bos E, Jeannerod M. Cognition. 2002; 85:177-187. [PubMed: 12127698]

9. Sun LD, Goldberg ME. Annu Rev Vis Sci. 2016; 2:61-84. [PubMed: 28532350]

10. Gross CG, Graziano MSA. Neuroscientist. 1995; 1:43-50. 
11. Andersen RA. Philos Trans R Soc Lond B Biol Sci. 1997; 352:1421-1428. [PubMed: 9368930]

12. Experiments involving brain-damaged patients and rodents cast doubt on this simplistic division. In fact, the hippocampus may primarily support egocentric, autonoetic views, whereas allocentric, semantic information is handled by the neocortex.

13. Rosenbaum RS, et al. Neuropsychologia. 2005; 43:989-1021. [PubMed: 15769487]

14. O'Keefe, J., Nadel, L. The Hippocampus as a Cognitive Map. Oxford Univ. Press; 1978.

15. Moser EI, et al. Nat Rev Neurosci. 2014; 15:466-481. [PubMed: 24917300]

16. Taube JS. Prog Neurobiol. 1998; 55:225-256. [PubMed: 9643555]

17. McNaughton BL, et al. J Exp Biol. 1996; 199:173-185. [PubMed: 8576689]

18. Eichenbaum H, Cohen NJ. Neuron. 2014; 83:764-770. [PubMed: 25144874]

19. Tulving E. Annu Rev Psychol. 2002; 53:1-25. [PubMed: 11752477]

20. Buzsáki G, Moser EI. Nat Neurosci. 2013; 16:130-138. [PubMed: 23354386]

21. Nadel L, Moscovitch M. Curr Opin Neurobiol. 1997; 7:217-227. [PubMed: 9142752]

22. Frankland PW, Bontempi B. Nat Rev Neurosci. 2005; 6:119-130. [PubMed: 15685217]

23. Pastalkova E, Itskov V, Amarasingham A, Buzsáki G. Science. 2008; 321:1322-1327. [PubMed: 18772431]

24. Eichenbaum H. Nat Rev Neurosci. 2014; 15:732-744. [PubMed: 25269553]

25. Wang Y, Romani S, Lustig B, Leonardo A, Pastalkova E. Nat Neurosci. 2015; 18:282-288. [PubMed: 25531571]

26. Kraus BJ, et al. Neuron. 2015; 88:578-589. [PubMed: 26539893]

27. Aronov D, Nevers R, Tank DW. Nature. 2017; 543:719-722. [PubMed: 28358077]

28. Fujisawa S, Amarasingham A, Harrison MT, Buzsáki G. Nat Neurosci. 2008; 11:823-833. [PubMed: 18516033]

29. Harvey CD, Coen P, Tank DW. Nature. 2012; 484:62-68. [PubMed: 22419153]

30. Church RM. Ann N Y Acad Sci. 1984; 423:566-582. [PubMed: 6588815]

31. Finnerty GT, Shadlen MN, Jazayeri M, Nobre AC, Buonomano DV. J Neurosci. 2015; 35:1391213916. [PubMed: 26468192]

32. Gibbon J, Malapani C, Dale CL, Gallistel C. Curr Opin Neurobiol. 1997; 7:170-184. [PubMed: 9142762]

33. Buonomano, D. Your Brain Is a Time Machine: The Neuroscience and Physics of Time. W. W. Norton; 2017.

34. Geisler C, Robbe D, Zugaro M, Sirota A, Buzsáki G. Proc Natl Acad Sci USA. 2007; 104:8149_ 8154. [PubMed: 17470808]

35. Honma M, Kuroda T, Futamura A, Shiromaru A, Kawamura M. Sci Rep. 2016; 6:25421. [PubMed: 27146904]

36. Eagleman DM, et al. J Neurosci. 2005; 25:10369-10371. [PubMed: 16280574]

37. Matin L, Pearce DG. Science. 1965; 148:1485-1488. [PubMed: 17738160]

38. Sommer MA, Wurtz RH. Nature. 2006; 444:374-377. [PubMed: 17093408]

39. Morrone MC, Ross J, Burr D. Nat Neurosci. 2005; 8:950-954. [PubMed: 15965472]

40. Perception of numerosity is also distorted by saccades, leading to the suggestion that parietal neurons code for magnitude rather than space or time.

41. Walsh V. Trends Cogn Sci. 2003; 7:483-488. [PubMed: 14585444]

42. DeLong AJ. Science. 1981; 213:681-683. [PubMed: 7256273]

43. Bonasia K, Blommesteyn J, Moscovitch M. Hippocampus. 2016; 26:9-12. [PubMed: 26418606]

44. Buzsáki G. Hippocampus. 2015; 25:1073-1188. [PubMed: 26135716]

45. Dennett DC, Kinsbourne M. Behav Brain Sci. 1992; 15:183-201.

46. Friston K, Buzsáki G. Trends Cogn Sci. 2016; 20:500-511. [PubMed: 27261057]

47. Bergson, H. Duration and Simultaneity. Durie, R., editor. Clinamen Press; 1999.

48. Lashley, KS. The problem of serial order in behavior. In: Jeffress, LA., editor. Cerebral Mechanisms in Behavior. Wiley; 1951. p. 112-136. 
49. Llinas, R. I of the Vortex: From Neurons to Self. MIT Press; 2001.

50. After ambulatory calibration of the distance metric, egocentric distance may be extracted from the spiking of binocular disparity-selective neurons in the primary visual cortex and the posterior parietal cortex.

51. Pouget A, Sejnowski TJ. Cereb Cortex. 1994; 4:314-329. [PubMed: 8075535]

52. Buzsáki G, Peyrache A, Kubie J. Cold Spring Harb Symp Quant Biol. 2014; 79:41-50. [PubMed: 25752314]

53. Reynolds JH, Heeger DJ. Neuron. 2009; 61:168-185. [PubMed: 19186161]

54. Teyler TJ, DiScenna P. Behav Neurosci. 1986; 100:147-154. [PubMed: 3008780]

55. Dede AJ, Frascino JC, Wixted JT, Squire LR. Proc Natl Acad Sci USA. 2016; 113:13480-13485. [PubMed: 27821761]

56. Fortin NJ, Agster KL, Eichenbaum HB. Nat Neurosci. 2002; 5:458-462. [PubMed: 11976705]

57. Hassabis D, Kumaran D, Vann SD, Maguire EA. Proc Natl Acad Sci USA. 2007; 104:1726-1731. [PubMed: 17229836]

58. Schacter DL, Addis DR, Buckner RL. Nat Rev Neurosci. 2007; 8:657-661. [PubMed: 17700624]

59. Amaral, D., Lavenex, P. Hippocampal neuroanatomy. In: Andersen, P.Morris, R.Amaral, D.Bliss, T., O'Keefe, J., editors. The Hippocampus Book. Oxford Univ. Press; 2006. p. 37-114.

60. Terada S, Sakurai Y, Nakahara H, Fujisawa S. Neuron. 2017; 94:1248-1262.e4. [PubMed: 28602691]

61. Such an "outside-in" approach has yielded a long list of ideas about the functions of the parietal cortex, including motor control, decision-making, attention, navigation, reward evaluation, topological relationships, higher-order processing, generation of body schema, mental rotation, episodic memory, numerosity, and imagination. A much longer list of hypotheses has been postulated for the function of the hippocampal system.

62. Nitz, DA. Space, Time and Memory in the Hippocampal Formation. Derdikman, D., Knierim, JJ., editors. Springer; 2014. p. 27-54. 

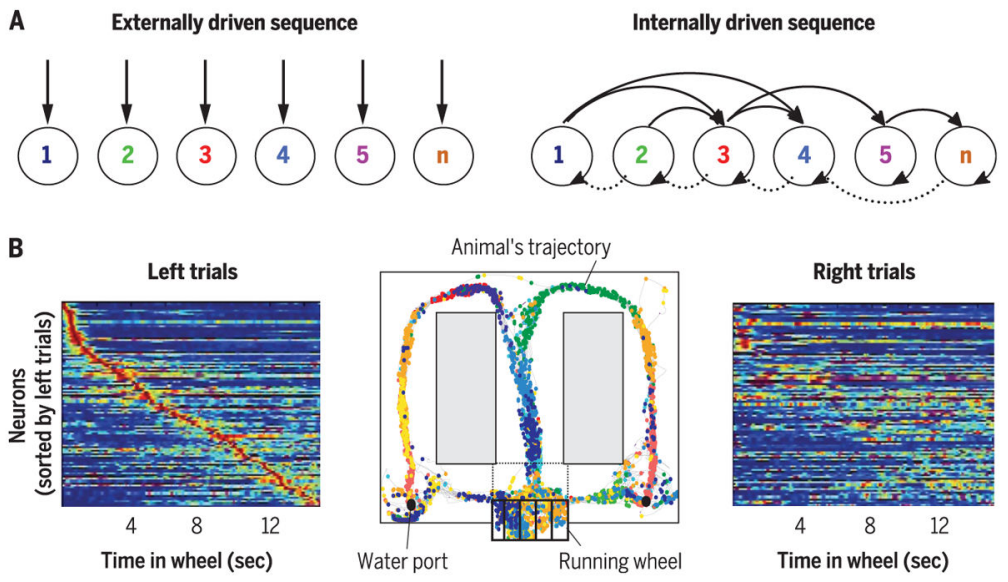

Fig. 1. Cell assembly sequences can track distance and duration

(A) During physical travel, successive assemblies of neurons (1 to $n$ ) respond sequentially because of the changing constellation of environmental landmarks and/or proprioceptive information from the body (left). During mental travel, sequential activation is supported by self-organized patterning (right). (B) Sequential activation of neuronal assemblies in an episodic memory task. (Middle) A rat was required to run in a running wheel during the delay between choosing either the left or right arms of the maze and to remember the last corridor choice. The rat obtained a water reward if it chose the arm opposite of the previous choice. Color-coded dots represent spike occurrences of simultaneously recorded hippocampal neurons. (Left) Normalized firing-rate profiles of neurons during wheel running, ordered by the latency of their peak firing rates during left trials (each line represents a single cell). (Right) Normalized firing rates of the same neurons during right trials. Note that an observer can infer the run duration (and distance) in the wheel from the sequential firing patterns of the neurons. Modified from (23). 


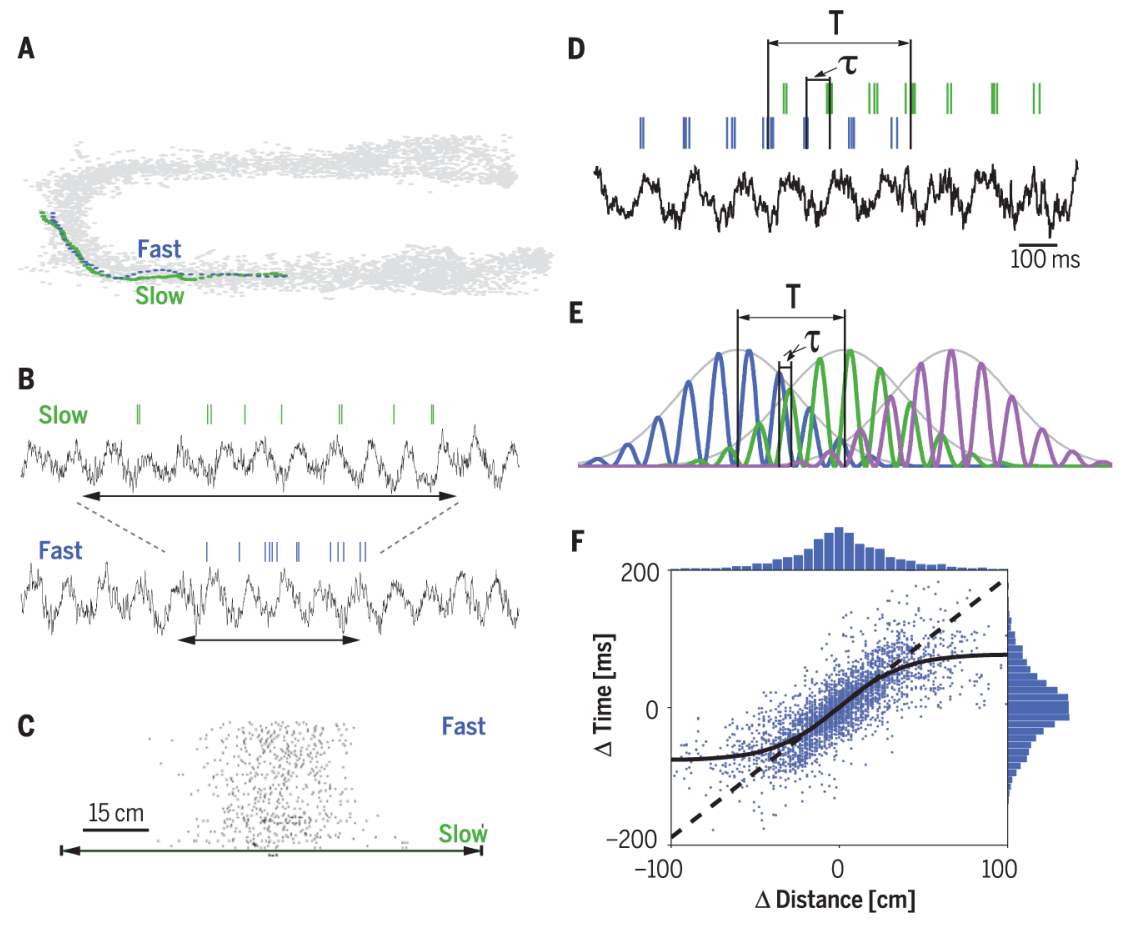

Fig. 2. Displacement-duration conversion by velocity in the hippocampus

(A) Trajectories of a rat through a place field on two trials with different speeds. (B) Spikes of one place cell and the corresponding local field potential (LFP) theta rhythm of the same two trials as in (A). Horizontal arrows indicate the time it took for the rat to run through the place field. (C) The number of spikes within the neuron's place field is similar on slow- and fast-run trials. Trials are sorted by velocity. (D) Spiking activity of two place cells (blue and green ticks) and LFP theta rhythm in a single run. Temporal duration (T) is the time needed for the rat to run the distance between the peaks of the two place fields (behavioral time scale). $\tau$, time offset between the two neurons within the theta cycle (theta time scale). (E) Three idealized place cells with identical theta oscillation frequency, illustrating the relationship between $\mathrm{T}$ and $\tau$. (F) Correlation between the distances of place field peaks and theta time scale lags $(\tau)$ for many pairs of neurons. Solid curve, sigmoid fit to the values; dashed line, line of equity. Above and right: histograms of distance and time lag, respectively. Modified from (34). 

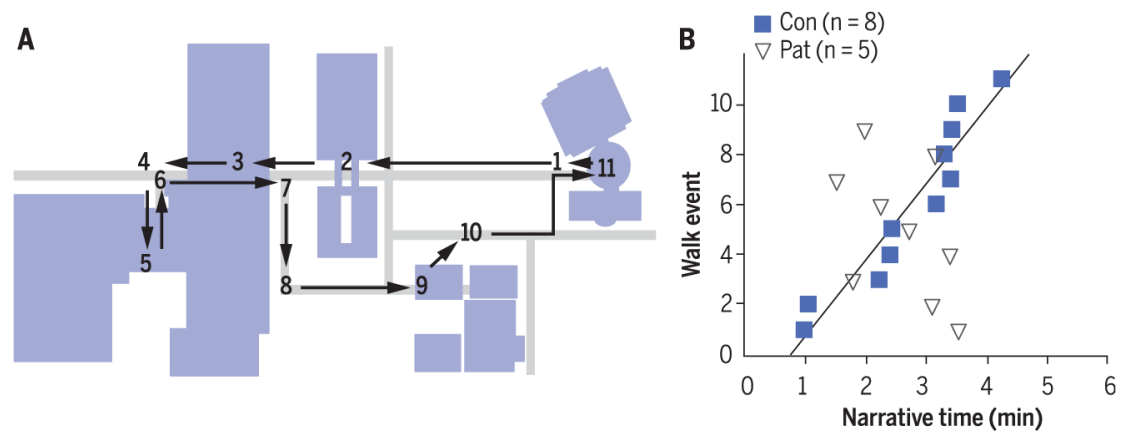

Fig. 3. The core deficit in patients with hippocampal lesions is their inability to narrate events in the order in which they occurred

(A) Map of 11 events that occurred during a guided campus walk. Sidewalks, gray; buildings, blue. Arrows indicate the path taken during the walk. (B) Events from the walk, described during 6-min narratives. The control group (Con; blue squares) tended to describe all 11 events in the order in which they occurred. The order in which the patients (Pat; open triangles) described events was unrelated to the order in which the events occurred. n, number of individuals in each group. Reproduced from (55). 